\title{
Hyperplasia of Myocyte Nuclei in Long-Term Cardiac Hypertrophy in Rats
}

\author{
Giorgio Olivetti, Roberto Ricci, and Piero Anversa \\ Departments of Pathology, University of Parma, Parma 43100, Italy; and New York Medical College, Valhalla, New York 10595
}

\begin{abstract}
In contrast to observations made in the human heart, hyperplasia of myocyte nuclei has never been demonstrated in experimental cardiac hypertrophy. To test the hypothesis that the duration of the mechanical load more than the magnitude of ventricular hypertrophy may be the inciting stimulus for myocyte nuclei hyperplasia, constriction of the pulmonary artery was produced in rats and the hearts were examined 6 mo later. A $76 \%$ increase in right ventricular weight was measured. This hypertrophic response was accompanied by a $41 \%$ increase in the total number of myocyte nuclei in the ventricle. Furthermore, average myocyte cell volume per nucleus increased by $\mathbf{2 8 \%}$. No changes in weight, myocyte size, and nuclear number were observed in the left ventricle. In conclusion, myocyte nuclear hyperplasia and cellular hypertrophy both participate to the adaptive response of the right ventricular myocardium in long-standing pressure overload cardiac hypertrophy.
\end{abstract}

\section{Introduction}

Experimental studies have demonstrated that short-term cardiac hypertrophy in the adult heart results from an enlargement of preexisting myocytes with a little DNA synthesis in myocyte nuclei, possibly representing polyploid cells $(1,2)$. Thus, it has become a general belief that no division of muscle nuclei occurs after a stimulus to ventricular growth once cell division has ceased, shortly after birth in the mammalian myocardium (2). In contrast, myocyte nuclei proliferation has been documented in humans under a variety of pathological conditions that impose a large and prolonged stress on the myocardium (3-5). A critical heart weight of $500 \mathrm{~g}$ has been considered the upper limit beyond which hyperplasia of nuclei in myofibers begins (3). These contrasting observations have raised a number of questions concerning, on one side, the validity of the conclusions drawn in humans, and, on the other, the difficulty of obtaining experimentally, degrees of hypertrophy comparable with those found in man. The critical heart weight theory, however, has neglected the role of time as a possibly important determinant of the cellular mechanisms implicated in long-standing cardiac hypertrophy. In the present report, we have tested the hypothesis that the long-

Address reprint requests to Dr. G. Olivetti, Department of Pathology, University of Parma, Via Gramsci 14, 43100 Parma, Italy.

Received for publication 1 June 1987.

J. Clin. Invest.

(c) The American Society for Clinical Investigation, Inc.

$0021-9738 / 87 / 12 / 1818 / 04 \$ 2.00$

Volume 80, December 1987, 1818-1821 term effects of a slowly progressive pressure overload on the right ventricle of the rat heart may lead to hyperplasia of myocyte nuclei despite a relatively moderate increase in ventricular mass. The right ventricle was preferred to the left one to avoid the influence on cardiac hypertrophy of a rise in perfusion pressure of the coronary circulation.

\section{Methods}

The main pulmonary artery was constricted at 2 mo of age in 12 male Wistar rats weighing $\sim 230 \mathrm{~g}$. After anesthesia with fentanyl citrate and droperidol (Leptofen, Farmitalia Carlo Erba Laboratories, Milan, Italy) $(0.1 \mathrm{ml}$ per $\mathrm{kg}$ of body weight, via the tail vein), a midsternal thoracotomy was performed and mechanical ventilation instituted with a small-animal respirator (501918; Ealing Corp., South Natick, MA). Under an operating microscope, the pulmonary artery was dissected free and a probe of $1.7 \mathrm{~mm}$ in diameter was held in contact with the vessel wall. The entire vessel and the probe was ligated with a suture (4-0), and then the probe was removed leaving a constricted opening in the lumen equal to the diameter of the probe. A small catheter connected to a negative pressure system was inserted in the pleural cavity to reduce the pneumothorax and allow the drainage of fluids, while the chest was closed. The intrathoracic catheter was removed a day after surgery. Nine sham-operated (SO) ${ }^{1}$ control rats were similarly treated, except that the ligature was not tied.

All animals were killed 6 mo later and the hearts were fixed by perfusion of the coronary vasculature with a solution containing $2 \%$ paraformaldehyde and $2.5 \%$ glutaraldehyde (6). The weights of the right and left ventricles were recorded and ventricular volumes computed by dividing ventricular weight by the specific gravity of muscle tissue, $1.06 \mathrm{~g} / \mathrm{ml}$ (7). The luminal diameter of the pulmonary artery in banded (B) and SO animals was measured at the level of the ligature utilizing a dissecting microscope with an ocular micrometer accurate to $0.05 \mathrm{~mm}$. Maximal and minimal internal diameters were determined at a magnification of 40 and the geometric mean value was calculated (8).

For the quantitative analysis of the myocardium, 20 specimens of the right and left ventricles from each heart were postfixed in osmium tetroxide and embedded in araldite (6). Ten randomly chosen tissue blocks from each ventricle were sectioned at a thickness of $0.75 \mu \mathrm{m}$ and stained with methylene blue and safranin. Morphometric sampling at a magnification of 1,000 consisted of counting the number of myocyte nuclear profiles, $N(n)$, in a measured area, $A$, of tissue sections in which cardiac muscle fibers were cut transversely. A square tissue area of $10,036 \mu \mathrm{m}^{2}$ was delineated in the microscopic field by an ocular reticle containing 42 sampling points (105844; Wild Heerbrugg Instruments, Inc., Farmingdale, NY). A total of 30 such fields were evaluated in each ventricle of each animal to determine the number of nuclear profiles per unit area of myocardium, $N(n)_{A}$, and the volume fraction of myocytes in the myocardium, $V(m)_{V}(9)$.

Nuclear length, $\bar{D}(n)$, was determined in each ventricle of each animal from 50 measurements made at a magnification of 1,250 in

1. Abbreviations used in this paper: B, banded; SO, sham operated. 
longitudinally oriented myocytes viewed with a microscope having an ocular micrometer accurate to $0.5 \mu \mathrm{m}$. Five blocks with myofibers sectioned perpendicular to their length were cut, sections $\sim 2 \mu \mathrm{m}$ in thickness collected and stained, and 10 measurements of nuclear length were recorded from each tissue section (9).

From the estimation of $N(n)_{A}$ and $\bar{D}(n)$, the number of myocyte nuclei per unit volume of myocardium, $N(n)_{V}$, was computed using the equation (9):

$$
N(n)_{V}=\frac{N(n)_{A}}{\bar{D}(n)} .
$$

Myocyte cell volume per nucleus, $\bar{V}(m)_{n}$, was obtained from the volume fraction of myocytes, $V(m)_{V}$, divided by the number of myocyte nuclei per unit volume of myocardium:

$$
\bar{V}(m)_{n}=\frac{V(m)_{V}}{N(n)_{V}} .
$$

The total number of myocyte nuclei in each ventricle, $N(n)_{T}$, was then derived from the product of the number per unit volume, $N(n)_{V}$, and the total ventricular volume, $V_{T}$ :

$$
N(n)_{T}=N(n)_{V} \cdot V_{T} .
$$

All morphometric data were collected blind and the code was broken at the end of the experiment. Results are expressed as mean $\pm \mathrm{SE}$ of values determined for the individual animals in each group. Statistical significance was determined using the unpaired two-tailed Student's $t$ test. $P$ values of $<0.05$ were considered to be significant.

\section{Results}

The surgical procedure employed resulted over a period of 6 mo in a $40 \%$ reduction in luminal diameter of the pulmonary artery from a value of $2.30 \pm 0.07$ to $1.37 \pm 0.09 \mathrm{~mm}(P$ $<0.0001$ ). During this interval right ventricular weight (Fig. 1 a) increased by $76 \%(P<0.005)$ with no change in the weight of the left ventricle inclusive of the septum (SO, 1,000 $\pm 43 \mathrm{mg}$; B, $950 \pm 48 \mathrm{mg}$ ). Right ventricular weight-to-body weight ratio (Fig. $1 b)$ increased by $93 \%(P<0.0001)$ since a $9 \%$ smaller body weight $(P<0.05)$ was found in B rats $(472 \pm 13 \mathrm{~g})$ with respect to $S O$ controls $(520 \pm 16 \mathrm{~g})$.
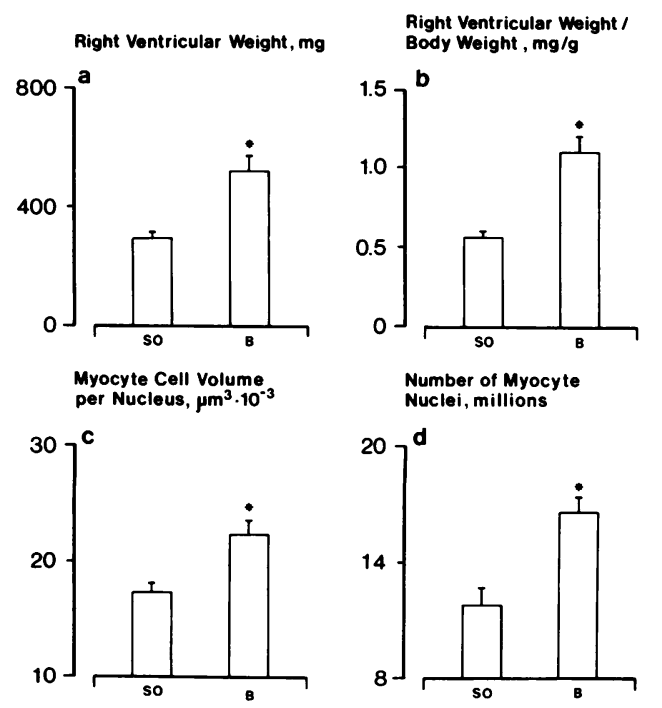

Figure 1. Effects of pulmonary artery banding on the right ventricular myocardium. * Indicates a statistically significant difference $(P$ $<0.05$ ) between $S O$ and pulmonary artery B rats.
The hypertrophic growth of the right ventricle was characterized by the absence of statistically significant changes in the numerical density of myocyte nuclei per millimeter squared of tissue (SO, 582 $\pm 22 \mathrm{mg}$; B, $501 \pm 34 \mathrm{mg}$ ). Furthermore, the volume fraction of myocytes in the myocardium remained practically constant after pulmonary artery stenosis (SO, $73.62 \pm 1.19 \%$; B, 75.96 $\pm 1.16 \%$ ). These data indicate that, despite a $76 \%$ increase in ventricular weight, the volume composition of the myocardium was not altered by the hypertrophic process. As expected, the lack of hypertrophy in the left ventricle was accompanied by comparable numbers of myocyte nuclear profiles per unit area of tissue in SO $(488 \pm 22 \mathrm{~g})$ and B $(513 \pm 22 \mathrm{~g})$ animals. The volume percent of myocytes was also nearly identical in the two animal groups (SO, 79.77 $\pm 0.86 \%$; B, 81.64 $0.63 \%$ ).

The average length of myocyte nuclei was $13.53 \pm 0.38$ and $13.91 \pm 0.31 \mu \mathrm{m}$ in the right ventricle of SO and B rats, respectively. Corresponding values in the left ventricle were $14.19 \pm 0.42$ and $15.14 \pm 0.34 \mu \mathrm{m}$. In the right ventricle, a $17 \%$ decrease in the numerical density of myocyte nuclei per millimeter cubed of myocardium was found in B animals $(36,100 \pm 2,500)$ with respect to SO controls $(43,400 \pm 2,200)$. This difference, however, was not statistically significant $(P$ $<0.1$ ). Values for the left ventricle were: SO, 34,600 $\pm 1,700$; B, $34,000 \pm 1,300$.

At all stages of growth cardiac myocytes of rats are composed of two populations, mononucleate and binucleate cells (10). This phenomenon complicates the quantitative analysis of tissue growth in terms of the fundamental processes of cellular hypertrophy and hyperplasia. Such analysis can be achieved for a mixed population, however, by introducing the more general concept of the mean cell volume per nucleus (11). Comparison of different values of myocyte cell volume per nucleus provides a direct measure of the growth of cellular components per nucleus, which is identical to cellular hypertrophy in a mononucleated population (11). This analysis demonstrated that right ventricular hypertrophy was accompanied by a $28 \%$ enlargement $(P<0.02)$ of the average myocyte cell volume per nucleus (Fig. $1 c$ ). Myocyte size in the left ventricle was similar in SO $\left(23,450 \pm 1,100 \mu \mathrm{m}^{3}\right)$ and B $\left(23,000 \pm 750 \mu \mathrm{m}^{3}\right)$ animals.

Regardless of the distribution of myocyte nuclei in single or multinucleate cells, a $41 \%$ increase $(P<0.0001)$ in the aggregate number of myocyte nuclei was found in the hypertrophied right ventricle of $\mathrm{B}$ rats (Fig. $1 d$ ) when compared with SO controls. The total number of myocyte nuclei in the left ventricle remained constant in the two animal groups (SO, $32.43 \pm 1.17$ millions; $B, 31.42 \pm 0.91$ millions).

\section{Discussion}

The results of the present study indicate that a moderate reduction in luminal diameter of the pulmonary artery in rats leads in 6 mo to a $76 \%$ increase in the mass of the right ventricle. Ventricular hypertrophy was the result of a $41 \%$ increase in the absolute number of myocyte nuclei and a $28 \%$ enlargement of the mean myocyte cell volume per nucleus. Thus, nuclear hyperplasia and cellular hypertrophy both participate to the hypertrophic response of the myocardium in this animal model.

It is well established that once myocyte proliferation ceases by the age of weaning in the rat, both physiologic and induced 
myocardial growth occur principally through hypertrophy of myocytes (2). In contrast, a hyperplastic component has been observed in cardiac enlargement produced in neonatal animals by nutritional anemia (12) or after aortic constriction in rats at 3 wk of age (13). There is only one experimental documentation of myocyte nuclei hyperplasia in the adult rat heart found in hypertrophied atrial tissue early after infarcts of the left ventricle (14). The differential adaptation between atrial and ventricular cells was attributed to the lower percentage of binucleated myocytes in the normal atrium (14). Binucleation appeared to be the limiting factor in nuclear proliferation in adult cardiac muscle cells. The present observations, however, demonstrate that, in spite of the fact that $\sim 90 \%$ of ventricular myocytes are binucleated in the rat heart $(2,10)$, nuclear hyperplasia does occur and may be regarded as an important compensatory mechanism of long-term cardiac hypertrophy.

Whether nuclear hyperplasia corresponds to an effective increase in cell number cannot be established at present. The proportion of mononucleated and multinucleated cells in the myocardium is not readily obtainable from quantitative analysis of tissue sections (11), complicating the computation of absolute cell number in the ventricle. It should also be pointed out that an identical caution applies to the previous quantitative estimations of cell size and number performed in human hearts (3-5).

In recent years, other methodologies have been described for the evaluation of myocyte size and number in myocardial tissue, through preparations of enzymatically dissociated myocytes $(10,15-17)$. With this technique, the frequency of different cell populations, based on the number of nuclei per cell (10), has been partially elucidated. However, this procedure cannot be used for the measurements of cell volume and number in the same heart because it has three severe limitations. First, cell size cannot be obtained in a reliable and accurate manner. As a result of flattening and spreading of the isolated cells when placed on the surface of a microscope slide, the mean myocyte cell volume is grossly overestimated (16, 17). Second, the percentage of myocardium constituted by parenchymal cells cannot be determined with this approach. This information is essential for the computation of the aggregate volume of the myocyte compartment in the entire ventricle. Such a value can then be divided by the average cell volume to yield the total number of cells in the whole ventricular tissue. Furthermore, the volume composition of the myocardium cannot be assumed to be constant, because the quantities of muscle cells and other tissue constituents may vary signifcantly during pathologic processes characterized by cell loss and scar formation within the myocardium $(9,18)$. Third, smaller mononucleated myocytes or larger multinucleated cells may be preferentially preserved during isolation, so that the partial sample obtained may not be representative of the actual proportion of cell populations in the tissue. Because of these multiple limitations, the in situ morphometric approach has been preferred to the cell isolation technique, despite the fact that changes in nuclear number cannot necessarily be translated into changes in myocyte cell number.

Myocardial hypertrophy in the rat, induced by different experimental procedures, consists at the most of an increase in weight of the affected ventricle of $\sim 80 \%$ (9). This growth response has been found to occur as early as $8 \mathrm{~d}$ after constriction of the abdominal aorta (19). Longer time intervals up to 2 mo after the imposition of an increased work load have not produced a greater magnitude of cardiac hypertrophy $(9,20$, 21). This is confirmed by the results obtained in the present study in which a 76\% ventricular hypertrophy was attained 6 mo after pulmonary artery banding. Thus, time may not influence the overall extent of myocardial hypertrophy, but it seems to play an important role on the growth mechanisms of the myocyte population in the enlarging myocardium. Although the sequence of events implicated in the transition from the phase of myocyte enlargement alone to that of nuclear proliferation was not investigated here, our observations strongly suggest that, like in humans (3-5), long-term myocardial hypertrophy evokes a hyperplastic response of cardiac muscle cell nuclei in the rat right ventricle.

In conclusion, we have provided the first experimental documentation that nuclear hyperplasia can occur in adult ventricular myocytes of the rat heart confirming the observations made in humans in the early sixties (3). Moreover, our findings suggest that this cellular process has to be regarded as a late event in response to a sustained mechanical stress more than a compensatory mechanism that takes place in the presence of a defined degree of cardiac hypertrophy.

\section{Acknowledgments}

The authors acknowledge the expert technical assistance of Miss Clara Bosco, Miss Claudia Catellani, Miss Costanza Lagrasta, Miss Lorraine Ostrubak, and Mr. Marco Visconti.

This work was supported by grant HL-38132 from the National Institutes of Health, and by a grant from the Consiglio Nazionale delle Ricerche.

\section{References}

1. Grove, D., R. Zak, K. G. Nair, and V. Aschenbrenner. 1969. Biochemical correlates of cardiac hypertrophy. IV. Observations on the cellular organizations of growth during myocardial hypertrophy in the rat. Circ. Res. 25:473-485.

2. Rakusan, K. 1984. Cardiac growth, maturation and aging. In Growth of the Heart in Health and Disease. R. Zak, editor. Raven Press, New York. 131-164.

3. Linzbach, A. J. 1960. Heart failure from the point of view of quantitative anatomy. Am. J. Cardiol. 5:370-382.

4. Astorri, E., A. Chizzola, O. Visioli, P. Anversa, G. Olivetti, and L. Vitali-Mazza. 1971. Right ventricular hypertrophy: a cytometric study on 55 human hearts. J. Mol. Cell. Cardiol. 2:99-1 10.

5. Astorri, E., R. Bolognesi, B. Colla, A. Chizzola, and O. Visioli. 1977. Left ventricular hypertrophy: a cytometric study on 42 human hearts. J. Mol. Cell. Cardiol. 9:763-775.

6. Anversa, P., C. Beghi, V. Levicky, S. L. McDonald, and Y. Kikkawa. 1982. Morphometry of right ventricular hypertrophy induced by strenuous exercise in rat. Am. J. Physiol. 243:H856-H861.

7. Mendez, J., and A. Keys. 1960. Density and composition of mammalian muscle. Metabolism. 9:184-188.

8. Olivetti, G., P. Anversa, M. Melissari, and A. V. Loud. 1980. Morphometric study of early postnatal development of the thoracic aorta in the rat. Circ. Res. 47:417-424.

9. Anversa, P., C. Beghi, Y. Kikkawa, and G. Olivetti. 1986. Myocardial infarction in rats. Infarct size, myocyte hypertrophy, and capillary growth. Circ. Res. 58:26-37.

10. Katzberg, A. A., B. B. Farmer, and R. A. Harris. 1977. The predominance of binucleation in isolated rat heart myocytes. Am. J. Anat. 149:489-500.

11. Anversa, P., G. Olivetti, and A. V. Loud. 1980. Morphometric study of early postnatal development in the left and right ventricular 
myocardium of the rat. I. Hypertrophy, hyperplasia, and binucleation of myocytes. Circ. Res. 46:495-502.

12. Neffgen, J. F., and B. Korecky. 1972. Cellular hyperplasia and hypertrophy in cardiomegalies induced by anemia in young and adult rats. Circ. Res. 30:104-113.

13. Dowell, R. T., and R. E. MacManus. 1978. Pressure-induced cardiac enlargement in neonatal and adult rats. Left ventricular functional characteristics and evidence of cardiac muscle cell proliferation in the neonate. Circ. Res. 42:303-310.

14. Oberpriller, J. W., V. J. Ferrans, and R. J. Carroll. 1983. Changes in DNA content, number of nuclei and cellular dimensions of young rat atrial myocytes in response to left coronary artery ligation. $J$. Mol. Cell. Cardiol. 15:31-42.

15. Bishop, S. P., S. Oparil, R. Reynold, and J. L. Drummond. 1979. Regional myocyte size in normotensive and spontaneously hypertensive rats. Hypertension (Dallas). 1:378-383.

16. Sorenson, A. L., D. Tepper, E. H. Sonnenblick, T. F. Robinson, and J. M. Capasso. 1985. Size and shape of enzymatically isolated ventricular myocytes from rats and hamsters. Cardiovasc. Res. 19:793-799.

17. Gerdes, A. M., J. A. Moore, J. M. Hines, P. A. Kirkland, and S. P. Bishop. 1986. Regional differences in myocyte size in normal rat heart. Anat. Rec. 215:420-426.

18. Olivetti, G., R. Ricci, C. Beghi, G. Guideri, and P. Anversa. 1986. Response of the border zone to myocardial infarction in rats. Am. J. Pathol. 125:476-483.

19. Anversa, P., G. Olivetti, M. Melissari, and A. V. Loud. 1980. Stereological measurement of cellular and subcellular hypertrophy and hyperplasia in the papillary muscle of adult rat. J. Mol. Cell. Cardiol. 12:781-795.

20. Hatt, P. Y., K. Rakusan, P. Gastineau, and M. Laplace. 1979. Morphometry and ultrastructure of heart hypertrophy induced by chronic volume overload (aorto-caval fistula in the rat). J. Mol. Cell. Cardiol. 11:989-998.

21. Julian, F. J., D. L. Morgan, R. L. Moss, M. Gonzales, and P. Dwivedi. 1981. Myocyte growth without physiological impairment in gradually induced rat cardiac hypertrophy. Circ. Res. 49:1300-1310. 